\title{
Chest Trauma: Current Recommendations for Rib Fractures, Pneumothorax, and Other Injuries
}

\author{
Michelle Kim ${ }^{1}$ • James E. Moore ${ }^{2}$ \\ Published online: 15 January 2020 \\ (C) Springer Science+Business Media, LLC, part of Springer Nature 2020
}

\begin{abstract}
Purpose of Review This article provides an overview of the common and important chest injuries that the anesthesiologist may encounter in patients following trauma including blunt injury, pneumothorax, hemothorax, blunt aortic injury, and blunt cardiac injury.

Recent Findings Rib fractures are frequently associated with chest injury and are associated with significant pain and other complications. Regional anesthesia techniques combined with a multimodal analgesic strategy can improve patient outcomes and reduce complications. There is increasing evidence for paravertebral blocks for this indication, and the myofascial plane blocks are a popular emerging technique. Recent changes to recommended management of tension pneumothorax are also described. Summary Chest trauma is commonly encountered, and anesthesiologists have the potential to significantly improve morbidity and mortality in this group of patients.
\end{abstract}

Keywords Chest trauma - Thoracic trauma $\cdot$ Rib fractures $\cdot$ Pneumothorax $\cdot$ Tension pneumothorax

\section{Introduction}

Chest trauma resulting from blunt or penetrating mechanisms is common, occurring in approximately $10 \%$ of patients admitted to hospital following injury [1]. Patients suffering from chest trauma are at high risk of developing significant morbidity and mortality. This can be due to the injury itself or because of the development of secondary complications, such as respiratory failure and pneumonia.

The most common presentation of blunt chest trauma is rib fractures. This most commonly occurs at the posterior aspect of the rib, the weakest point. With the significant amount of force required to break ribs and the associated

This article is part of the Topical Collection on Anesthesia for Trauma

James E. Moore

james.moore@ccdhb.org.nz

Michelle Kim

michelle.kim@som.umaryland.edu

1 University of Maryland School of Medicine, R. Adams Cowley Shock Trauma Center, Baltimore, MD, USA

2 Consultant Anaesthetist, Intensive Care Physician \& Director of Trauma Services, Wellington Hospital, Wellington, New Zealand energy transfer, damage to underlying tissues occurs resulting in injuries such as pulmonary contusions. Optimal management of rib fractures can prevent complications and reduce associated mortality. The mainstay of this management includes pulmonary hygiene, early mobility, and, importantly, excellent analgesia, which anesthesiologists can directly influence.

Other common examples of chest injury encountered in anesthetic practice include pneumothorax, for which there have been some recent important changes to the recommended management, hemothorax, and injury to the heart and great vessels. In this review, we will provide an overview of the evidence and current recommendations for the management of these types of chest trauma, with a particular emphasis on pain management for rib fractures, and changes to the recommended management of tension pneumothorax.

\section{Rib Fractures}

The severity of chest injury is proportional to the number of ribs fractured. The baseline mortality for patients admitted to hospital following rib fractures is $10 \%$, but this increases with each additional rib fractured, approaching $40 \%$ if $>6$ ribs are fractured [1]. 


\section{Pain Management}

The chest wall is highly innervated by pairs of intercostal nerves traveling along the inferior border of each rib. As a result, rib fractures are associated with significant pain. Chest wall pain resulting from multiple rib fractures can exacerbate pulmonary complications associated with blunt thoracic injury. Significant and uncontrolled pain contributes to splinting, guarding, low tidal volumes, decreased mobility, insufficient ability to cough and clear secretions, and inability to participate in chest physiotherapy. These factors further impair the already compromised pulmonary system leading to complications such as atelectasis, pneumonia, acute respiratory distress syndrome (ARDS), and pulmonary embolism, effectively increasing the overall morbidity and mortality associated with blunt thoracic injury. Thus, the cornerstone of the management of patients with multiple rib fractures includes aggressive management of chest wall pain.

Close consideration of the effectiveness, safety, and side effect profile of an analgesic intervention is paramount in the selection of an appropriate management strategy. When treating the patient with multiple rib fractures, it is of particular importance to provide enough pain relief to allow for improvement of pulmonary mechanics, clearance of secretions, and patient mobility. A variety of approaches to pain management exist, which include systemic analgesia and regional techniques.

\section{Systemic Analgesia}

Historically, systemic opioids have been used as first-line agents to alleviate pain resulting from multiple rib fractures. Administration via oral, intravenous (intermittent, continuous, and patient-controlled analgesia), intramuscular, transdermal, and nebulized routes have all proven effective [2-7]. Mackersie et al. found that continuous infusion of intravenous fentanyl improved visual analog pain scores and vital capacity. However, respiratory depression and hypoxemia also ensued [3]. Systemic opioids, while potentially effective, are known to cause sedation, respiratory and cough suppression, and delirium [8].

The use of a multimodal systemic analgesic approach in treating patients with multiple rib fractures may offer the benefit of decreasing narcotic use and therefore reducing the risk of associated adverse effects. Further, by targeting distinct pain pathways, improved efficacy may also occur. Early administration of parenteral nonsteroidal anti-inflammatory drugs (NSAID) has been observed to decrease opioid requirements, pneumonia incidence, both hospital and ICU length of stay, and the number of ventilator days [9•, 10•]. However, there has been a paucity of data to suggest a clear benefit to using agents such as anticonvulsants, lidocaine patches, or ketamine. Nonetheless, according to the Eastern Association for the Surgery of Trauma (EAST) and Trauma Anesthesiology Society (TAS) joint practice management guideline for pain management for blunt thoracic trauma, the use of multimodal analgesia is conditionally recommended for adult patients with blunt thoracic trauma despite the low quality and limited evidence [11•]. Thus, it is reasonable to implement this mode of analgesia in lower-risk patients - age $<$ 65 years old, $<3$ rib fractures, no significant pulmonary compromise, and no comorbidities [1,12-14] - and in conjunction with a regional anesthetic technique.

\section{Regional Analgesia}

Regional analgesia offers a plethora of benefits in patients with multiple rib fractures. Pain relief is often satisfactory and immediate, with a minimal side effect profile. Figure 1 illustrates a number of options for regional techniques which will be discussed further below.

\section{Epidural}

Thoracic epidural analgesia (TEA) using local anesthetic with or without opioids provides excellent pain relief in patients with blunt thoracic trauma. Epidural analgesia has been shown to have particular benefit in terms of subjective pain score [3, 4]; improvement in respiratory parameters including FRC, lung compliance, $\mathrm{PaO} 2$, vital capacity; and decreased incidence of somnolence, gastrointestinal disturbance, and respiratory depression [15]. Moreover, the placement of TEA provides multilevel and bilateral coverage. Because of these advantages, TEA has traditionally been considered the gold standard regional technique for treating patients with multiple rib fractures. However, more recent data suggest that the benefits of utilizing TEA may not be as pronounced as was once believed.

A meta-analysis of randomized controlled trials (RCT) performed by Carrier et al. concluded that when compared to other analgesic interventions, epidural placement was not associated with improvement in mortality or ICU and hospital length of stay [16]. Several other recent meta-analyses and systematic reviews of RCTs have confirmed these findings $[17 \bullet, 18 \bullet, 19]$. Furthermore, differences in the duration of mechanical ventilation and pulmonary complications such as pneumonia and empyema have also been questioned [20 $]$. In fact, in a matched analysis examining over 1000 patients with traumatic rib fractures, McKendy et al. found that epidural analgesia was associated with an increase in pulmonary complications [21•]. Despite these findings, EAST/TAS guidelines currently recommend placement of epidural analgesia over non-regional modalities based on very low-quality evidence, emphasizing the importance of patient preference $[11 \bullet]$. 


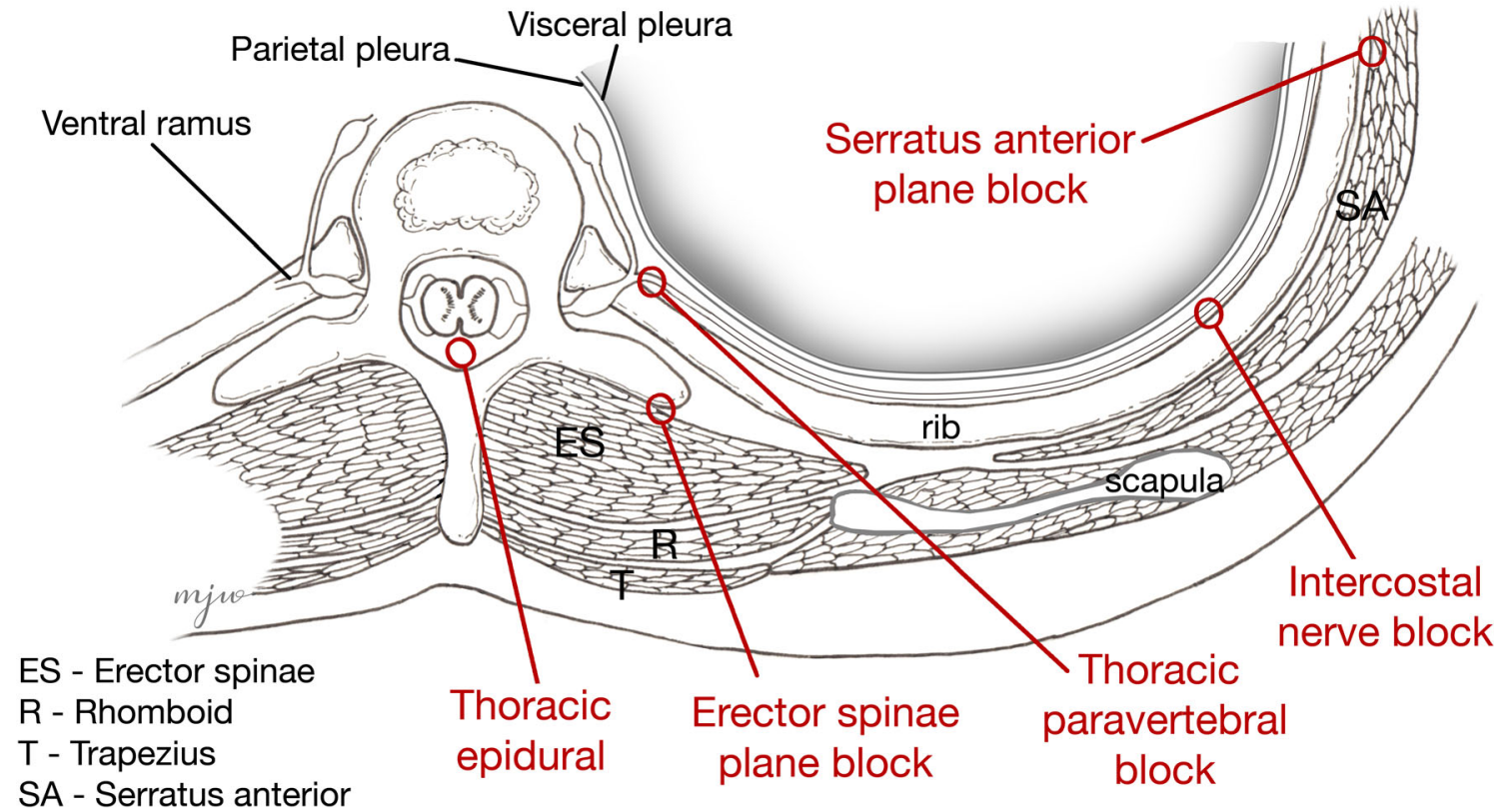

Fig. 1 Regional analgesia options for pain management in multiple rib fractures

It is critical to consider these recent data and recommendations for TEA, as well as its limitations when selecting an analgesic technique for the patient with multiple rib fractures. TEA is contraindicated in patients with hypotension, hypovolemia, coagulopathy, significant spinal or traumatic brain injuries, and systemic infection, all of which are common in the multi-trauma patient. Placement of TEA is particularly associated with hypotension, mainly due to a reduction in systemic vascular resistance from the blockade of sympathetic output. This can result in significant hemodynamic compromise, especially in the under-resuscitated patient. Moreover, the technically demanding nature of this procedure may pose challenges in a patient with multiple injuries and sites of pain. Finally, it is important to consider the risks of dural puncture, motor blockade, pruritis, and urinary retention.

\section{Thoracic Paravertebral Block}

Thoracic paravertebral blocks (TPVB) enable the administration of local anesthetic to ventral and dorsal rami of ventral nerves within the paravertebral space. Analgesia can be achieved by intermittent injection [22], continuous infusion [23], or regular dosing through a catheter [24], which can produce a unilateral multilevel nerve blockade in contiguous dermatomes [25, 26]. Many institutions have adapted TPVBs as a means to manage patients with multiple rib fractures because of its relative technical ease, liberal anticoagulation guidelines, lower incidence of sympathectomy, preservation of bladder sensation, and absent risk of spinal cord injury. Furthermore, since there is sparing of lumbar and sacral nerve roots, TPVB can be performed in patients who require neurological assessment for concomitant spinal cord compression [22, 27-29].

TPVB has proven to be as effective as TEA for pain management in patients with unilateral rib fractures [30] and may contribute to improved survival [20]. Further, when compared with patients receiving intravenous patient-controlled analgesia (PCA), patients with TPVB reported lower pain scores; demonstrated superior respiratory parameters including FVC, FEV1/FVC, $\mathrm{PaO} 2$, and $\mathrm{PaO} 2 / \mathrm{FiO} 2$; and experienced a lower incidence of nausea and vomiting [31•]. The complication rate is low and has been described as comparable to that of epidural and intercostal nerve blocks [32]. It can be argued that complications associated with TPVBs are less detrimental than those of neuraxial techniques. For example, hypotension that can occur with TPVBs requires less medical intervention than the expected sympathectomy associated with TEA. Nonetheless, there remains the risk of pneumothorax, pleural puncture, inadvertent bilateral blockade, and vascular puncture [28]. With the advent of ultrasound technology and its utility in more precise TPVB placement, TPVB may have an improved safety profile compared to TEA.

\section{Intercostal Nerve Block}

Intercostal nerve blocks (ICB) may also be utilized in patients with multiple rib fractures. This procedure is simple to perform and has a very localized area of effect. However, because of the segmental overlap of intercostal nerves, injection both above and below the affected rib(s) is required to produce adequate coverage. This necessitates multiple injections, which increases the risk for complications such as vascular puncture, pneumothorax, patient discomfort due to the need 
for palpation of the fractured rib during local anesthetic (LA) administration, and LA toxicity because of the high systemic absorption [33]. Furthermore, performing ICB for rib fractures in upper segments may prove challenging because of the presence of the scapula and rhomboid muscles at levels T1-T7.

While pain relief with ICB is often immediate and adequate, the effect of a single injection of LA typically lasts 48 h. Continuous intercostal nerve block catheters (CICNB) can theoretically provide analgesia for longer durations, and there is some evidence pointing to its utility in decreasing pain scores and decreasing hospital length of stay [34]. However, further studies are needed in this arena to fully understand the efficacy of CICNB for patients with multiple rib fractures.

\section{Myofascial Plane Blocks}

Advances in and the increasingly widespread use of ultrasound technology have contributed to improved safety and utility of regional anesthetic techniques. Additionally, the ability to identify anatomical landmarks in real time has popularized the use of alternative regional analgesia techniques such as myofascial plane blocks. In the context of rib fracture management, two innovative myofascial plane blocks have gained in popularity: serratus anterior and erector spinae plane blocks. These procedures offer technical simplicity with a favorable side effect profile while providing unilateral multilevel coverage. In comparison to other regional techniques, there is virtually no risk of hemodynamic changes, motor blockade, vascular penetrance, or inadvertent intrathecal injection. Additionally, both methods can be utilized in patients with impaired coagulation.

Serratus anterior plane blocks (SAP) are performed at the level of the fifth rib at the mid-axillary line, while the patient is sitting or supine. Ultrasound guidance can be used to identify the latissimus dorsi and serratus anterior muscles, and LA is injected either superficial or deep to the serratus anterior muscle. This provides analgesia to the lateral branches of the intercostal nerves T2-T9. Notably, SAP will provide coverage to the anterior two-thirds of the hemothorax only, limiting its utility to rib fractures at these locations. Several case reports have demonstrated a reduction in opioid use and improvement in pain scores after the performance of SAP in patients with multiple rib fractures [35-37].

Erector spinae plane blocks (ESP), on the other hand, provide analgesia to the anterior, lateral, and posterior hemothorax. Numerous case reports have identified its value in patients with multiple rib fractures [38-41]. Most recently, Adhikary et al. demonstrated a significant improvement in inspiratory volumes and a reduction in pain scores and opioid requirements [42•]. In order to perform this block, the patient should be positioned either seated or laterally. Ultrasound is used to identify the transverse process and the erector spinae muscle directly overlying the transverse process. LA injected deep to the erector spinae muscle results in a cephalocaudad spread of LA, providing widespread analgesia to the hemothorax.

Myofascial plane blocks have the potential to offer great utility in patients with multiple rib fractures. However, at present, additional studies are needed to further clarify their efficacy, safety, and local anesthetic dosing in patients with chest trauma.

\section{Rib Fixation Surgery}

Various techniques for rib fixation surgery have been described for almost 100 years, utilizing a variety of devices [43]. Recent advances in rib plating technology and an increase in commercially available rib specific fixation systems have invigorated interest in the procedure.

Benefits of rib fixation appear to be a reduction in duration of dependence on mechanical ventilation and decreased ICU and hospital length of stay [44, 45, 46॰], particularly in patients with early flail chest fixation (48 to $72 \mathrm{~h}$ ) post injury [47•]. However, current evidence suggests no change in longer-term pain outcomes or quality of life indicators, and in 2017, EAST only conditionally recommended rib fixation surgery to decrease mortality, hospital and ICU length of stay, incidence of pneumonia, and need for tracheostomy in light of low quality of evidence [46•].

Our current practice is to offer the procedure to mechanically ventilated patients in the ICU with respiratory failure felt to be predominately due to their multiple rib fractures and in the absence of a contraindication such as concomitant severe traumatic brain injury.

\section{Pneumothorax}

Pneumothorax is a common finding after trauma, and there is a spectrum of clinical presentations ranging from incidental findings of occult pneumothorax (visible only on advanced imaging such as computed tomography [CT]) through to the potentially lethal tension pneumothorax syndrome.

In general, a pneumothorax which is visible on plain chest X-ray following trauma should be treated with the placement of an intercostal drain. There has been a move toward smaller Seldinger-style intercostal drains for management of isolated spontaneous pneumothorax in the non-trauma population; however, traumatic pneumothoraces are commonly associated with associated bleeding, so in general, we would tend to place a 24-32Fr intercostal drain using a traditional surgical approach.

The widespread adoption of whole-body computed tomography following trauma (protocolized $\mathrm{CT}$ imaging of the chest, c-spine, chest, abdomen, and pelvis) has led to the identification of a group of so-called occult pneumothoraces which are only visible on CT imaging. The question of how to manage these occult pneumothoraces remains unresolved, 
especially if the patient requires positive pressure ventilation. However, conservative management with careful observation appears to be a reasonable approach, with intercostal catheter placement required in only $5-14 \%$ of patients $[48,49]$.

\section{Tension Pneumothorax}

Tension pneumothorax is a rapidly lethal condition where air enters the pleural space via with a one-way flap mechanism, gradually building up more and more positive pressure within the pleural space. Eventually, the positive pressure builds to the point where mediastinal structures shift toward the opposite hemothorax, and the pressure is so great that venous return to the right heart falls resulting in profound hypotension. The clinical presentation of tension pneumothorax can include severe dyspnea and air hunger in the awake patient, through to cardiovascular collapse (especially in mechanically ventilated patients). The presence of tension pneumothorax should be actively excluded in all trauma patients during the initial primary survey and should be considered in the differential diagnosis of any unanticipated deterioration of a trauma patient. The diagnosis should be made on clinical grounds with signs including absent or reduced breath sounds in the affected hemothorax; distended neck veins (noting that this may be absent if the patient has concurrent hypovolemic shock); tracheal deviation away from the affected hemothorax; of hyper-resonance, which may be difficult to demonstrate clinically but can be useful to differentiate from a massive hemothorax (another cause of unilateral absent air entry). Bedside trauma ultrasonography (extended focused assessment sonography for trauma, or E-FAST) is highly sensitive in the hands of an experienced clinician [50]; however, interpretation must always take into account the clinical context of the patient's presentation.

\section{Management}

The priority in the management of tension pneumothorax is decompression of the pleura. The traditional teaching has been needle decompression via the second intercostal space in the midclavicular line; however, there are several problems with this approach. Firstly, the overlying soft tissues in this location are such that a standard $5 \mathrm{~cm}$ cannula will not reach the pleura in up to $30 \%$ of patients [51, 52]. Secondly, in an emergency, clinicians frequently misjudge where the mid-clavicular line is and will tend to approach too medially, risking injury to major vascular structures and the internal mammary artery [53]. For these reasons, the Committee on Trauma of the American College of Surgeons has revised their recommendation in the Advanced Trauma Life Support (ATLS $®$ ) 10th edition [54] to suggesting the fourth or fifth intercostal space between the mid- and anterior axillary lines as the optimal site of decompression in adults (the previous second intercostal space recommendation remains for pediatric patients). They have also recommended finger thoracostomy as an alternative approach. Finger thoracostomy is similar to the initial steps in surgical intercostal drain placement: the skin is prepped and ideally anesthetized, a small $3-4 \mathrm{~cm}$ incision is made along the superior border of the rib, and a sterile gloved finger is introduced, bluntly dissecting until the pleural space is entered. Prompt placement of a formal intercostal drain should ideally follow.

Although ATLS® recommends both techniques, the authors' preference remains needle decompression as an initial emergency technique. However, one should be aware that, especially in larger patients, the needle may not adequately reach or decompress the pleura; therefore, you should prepared to undertake finger thoracostomy or rapid intercostal drain insertion if initial needle decompression is unsuccessful.

\section{Hemothorax}

Bleeding into the pleural space is also commonly observed in chest trauma. The most common causes are intercostal vessel injury associated with rib fractures, bleeding from injured lung tissue, or more rarely from sub-lobar, lobar, or great vessel injury. A large amount of bleeding into the pleura results in massive hemothorax, often defined as the presence of $>$ $1500 \mathrm{ml}$ blood on initial intercostal drain placement, or ongoing bleeding $>200 \mathrm{ml} / \mathrm{h}$ over the first 2 to $4 \mathrm{~h}$ [55]. This injury can result in a mixed form of shock with hypovolemia from bleeding, as well as an element of obstructive shock from impaired venous return due to the high pressure exerted within the pleural space (similar to a tension pneumothorax). Management involves the decompression of the pleura by the placement of an intercostal drain, with the concurrent restoration of volume utilizing a hemostatic resuscitative approach [56••]. Most patients with hemothorax can be managed conservatively with intercostal drain placement alone, but those with large volume or ongoing active bleeding may require operative intervention to control bleeding.

\section{Blunt Aortic Injury}

Sudden decelerative forces, such as those experienced during a high-speed motor vehicle crash or high fall, can lead to blunt aortic injury. The most common site is at the ligamentum arteriosum, just distal to the left subclavian artery, where the relatively mobile portion of the distal aortic arch meets the relatively fixed proximal descending thoracic aorta. This decelerative force can cause aortic transection (which is almost universally fatal at the scene), as well as intimal tears or pseudoaneurysms. Blunt aortic injury is most commonly identified during arterial phase $\mathrm{CT}$ imaging of the chest [57]. Traditionally, these would be primarily managed with blood pressure control; however, technological advances and 
increasing experience in the use of endoluminal stent grafts have led to endovascular management as the most common method of managing blunt aortic injury [58]. Given the significant energy transfer generally associated with blunt aortic injuries, other injuries are common (and in the case of the hypotensive patient, alternative injuries are the most likely cause rather than the aortic injury). Because blunt aortic injuries are frequently asymptomatic, a high index of suspicion based on the mechanism of injury should be maintained until ruled out by adequate $\mathrm{CT}$ imaging.

\section{Blunt Cardiac Injury}

Blunt cardiac injury can occur as the heart collides with the sternum and chest wall during sudden decelerative forces, such as being ejected from a motor vehicle. Although myocardial contusion is the most common manifestation of blunt cardiac injury, a range of injuries can occur, including myocardial rupture, valve rupture (leading to acute valvular regurgitation), and coronary artery dissection. It is also reasonably common for a primary medical event to lead to trauma, for instance, the elderly patient who crashes their car while suffering a myocardial infarct.

The concern is that myocardial contusion can develop and lead to arrhythmias and impaired myocardial function (or cardiogenic shock). The identification of patients with blunt cardiac injury can be challenging; one retrospective registry study has suggested an association with sternal fracture [59].

A normal serial 12-lead electrocardiogram in the context of a hemodynamically normal patient appears to be reassuring [60]. The role of biomarkers, specifically troponin, in the diagnosis of blunt cardiac injury remains unclear; however, troponin elevation in this setting is associated with worse outcomes $[61 \cdot]$.

Unexplained shock or hypotension in a patient who has sustained a high-energy decelerative force should prompt consideration of significant blunt cardiac injury. The most appropriate screening test in this situation is probably urgent transthoracic or transesophageal echocardiography.

\section{Conclusion}

Chest trauma is a common presentation following injury and is associated with significant morbidity and mortality. Rib fractures are a frequent occurrence and are a marker of energy transfer and underlying injury. Pain from rib fractures can result in impaired ventilation and oxygenation, so the mainstay of treatment is good quality analgesia. A plethora of regional analgesia options are available, all with associated benefits and risks. The optimal regional analgesia technique is as yet undefined, although there is increasing evidence for paravertebral catheters. Myofascial plane blocks have become increasingly popular and offer a favorable risk and side-effect profile, although the evidence supporting the efficacy of this technique is currently limited.

Tension pneumothorax remains an important and immediately life-threatening condition. The most recent edition of ATLS $®$ has recommended pleural decompression in the fourth to fifth intercostal space between the mid- and anterior axillary lines, utilizing either needle decompression or finger thoracostomy - an important change relevant to anesthesiologists who care for trauma patients. Anesthesiologists are wellplaced to be able to positively impact the course and outcome of this important group of patients.

Acknowledgments The authors wish to thank Dr. Jemimah Walker for her review of the manuscript, and Mark Weiber for producing the illustration.

\section{Compliance with Ethical Standards}

Conflict of Interest The authors declare that they have no conflict of interest.

Human and Animal Rights and Informed Consent This article does not contain any studies with human or animal subjects performed by any of the authors.

\section{References}

Papers of particular interest, published recently, have been highlighted as:

- Of importance

•. Of major importance

1. Flagel BT, Luchette FA, Reed RL, Esposito TJ, Davis KA, Santaniello JM, et al. Half-a-dozen ribs: the breakpoint for mortality. Surgery. 2005;138(4):717-23; discussion 23-5. https://doi.org/ 10.1016/j.surg.2005.07.022.

2. O'Kelly E, Garry B. Continuous pain relief for multiple fractured ribs. Br J Anaesth. 1981;53(9):989-91. https://doi.org/10.1093/bja/ 53.9.989.

3. Mackersie RC, Karagianes TG, Hoyt DB, Davis JW. Prospective evaluation of epidural and intravenous administration of fentanyl for pain control and restoration of ventilatory function following multiple rib fractures. J Trauma. 1991;31(4):443-9 discussion 9-51.

4. Moon MR, Luchette FA, Gibson SW, Crews J, Sudarshan G, Hurst JM, et al. Prospective, randomized comparison of epidural versus parenteral opioid analgesia in thoracic trauma. Ann Surg. 1999;229(5):684-91; discussion 91-2. https://doi.org/10.1097/ 00000658-199905000-00011.

5. Ravalia A, Suresh D. I.V. alfentanil analgesia for physiotherapy following rib fractures. Br J Anaesth. 1990;64(6):746-8. https:// doi.org/10.1093/bja/64.6.746.

6. Solak O, Oz G, Kokulu S, Solak O, Dogan G, Esme H, et al. The effectiveness of transdermal opioid in the management multiple rib fractures: randomized clinical trial. Balkan Med J. 2013;30(3):27781. https://doi.org/10.5152/balkanmedj.2013.8191.

7. Fulda GJ, Giberson F, Fagraeus L. A prospective randomized trial of nebulized morphine compared with patient-controlled analgesia 
morphine in the management of acute thoracic pain. J Trauma. 2005;59(2):383-8; discussion 9-90. https://doi.org/10.1097/01.ta. 0000179452.70520.1b.

8. Benyamin R, Trescot AM, Datta S, Buenaventura R, Adlaka R, Sehgal N, et al. Opioid complications and side effects. Pain Physician. 2008;11(2 Suppl):S105-20.

9. Bayouth L, Safcsak K, Cheatham ML, Smith CP, Birrer KL, Promes JT. Early intravenous ibuprofen decreases narcotic requirement and length of stay after traumatic rib fracture. Am Surg. 2013;79(11):1207-12 Evidence for NSAID use in rib fractures.

10. Yang Y, Young JB, Schermer CR, Utter GH. Use of ketorolac is associated with decreased pneumonia following rib fractures. Am J Surg. 2014;207(4):566-72. https://doi.org/10.1016/j.amjsurg.2013. 05.011 Evidence for reduced complications if NSAIDs used following rib fractures.

11. Galvagno SM Jr, Smith CE, Varon AJ, Hasenboehler EA, Sultan S, Shaefer G, et al. Pain management for blunt thoracic trauma: a joint practice management guideline from the Eastern Association for the Surgery of Trauma and Trauma Anesthesiology Society. J Trauma Acute Care Surg. 2016;81(5):936-51. https://doi.org/10.1097/TA. 0000000000001209 Recent combined society guidance on blunt trauma analgesia.

12. Battle CE, Hutchings H, Evans PA. Risk factors that predict mortality in patients with blunt chest wall trauma: a systematic review and meta-analysis. Injury. 2012;43(1):8-17. https://doi.org/10. 1016/j.injury.2011.01.004.

13. Bergeron E, Lavoie A, Clas D, Moore L, Ratte S, Tetreault S, et al. Elderly trauma patients with rib fractures are at greater risk of death and pneumonia. J Trauma. 2003;54(3):478-85. https://doi.org/10. 1097/01.TA.0000037095.83469.4C.

14. Bulger EM, Edwards T, Klotz P, Jurkovich GJ. Epidural analgesia improves outcome after multiple rib fractures. Surgery. 2004;136(2):426-30. https://doi.org/10.1016/j.surg.2004.05.019.

15. Simon BJ, Cushman J, Barraco R, Lane V, Luchette FA, Miglietta $\mathrm{M}$, et al. Pain management guidelines for blunt thoracic trauma. J Trauma. 2005;59(5):1256-67. https://doi.org/10.1097/01.ta. 0000178063.77946.f5.

16. Carrier FM, Turgeon AF, Nicole PC, Trepanier CA, Fergusson DA, Thauvette D, et al. Effect of epidural analgesia in patients with traumatic rib fractures: a systematic review and meta-analysis of randomized controlled trials. Can J Anaesth. 2009;56(3):230-42. https://doi.org/10.1007/s12630-009-9052-7.

17. Duch P, Moller MH. Epidural analgesia in patients with traumatic rib fractures: a systematic review of randomised controlled trials. Acta Anaesthesiol Scand. 2015;59(6):698-709. https://doi.org/10. 1111/aas.12475 Recent systematic review which questions the role of thoracic epidurals.

18. Peek J, Smeeing DPJ, Hietbrink F, Houwert RM, Marsman M, de Jong MB. Comparison of analgesic interventions for traumatic rib fractures: a systematic review and meta-analysis. Eur J Trauma Emerg Surg. 2019;45(4):597-622. doi:https://doi.org/10.1007/ s00068-018-0918-7. Review suggesting good analgesia with thoracic epidurals, but lack of improvement in other important endpoints.

19. Zaw AA, Murry J, Hoang D, Chen K, Louy C, Bloom MB, et al. Epidural analgesia after rib fractures. Am Surg. 2015;81(10):950-4.

20. Malekpour M, Hashmi A, Dove J, Torres D, Wild J. Analgesic choice in management of rib fractures: paravertebral block or epidural analgesia? Anesth Analg. 2017;124(6):1906-11. https://doi. org/10.1213/ANE.0000000000002113 Suggests equivalence between epidural \& paravertebral catheters.

21. McKendy KM, Lee LF, Boulva K, Deckelbaum DL, Mulder DS, Razek TS, et al. Epidural analgesia for traumatic rib fractures is associated with worse outcomes: a matched analysis. J Surg Res. 2017;214:117-23. https://doi.org/10.1016/j.jss.2017.02.057
Cohort study suggesting poorer outcomes in patients with epidural management following chest trauma.

22. Gilbert J, Hultman J. Thoracic paravertebral block: a method of pain control. Acta Anaesthesiol Scand. 1989;33(2):142-5. https:// doi.org/10.1111/j.1399-6576.1989.tb02877.x.

23. McKnight CK, Marshall M. Monoplatythela and paravertebral block. Anaesthesia. 1984;39(11):1147. https://doi.org/10.1111/j. 1365-2044.1984.tb08953.x.

24. Williamson S, Kumar CM. Paravertebral block in head injured patient with chest trauma. Anaesthesia. 1997;52(3):284-5.

25. Cheema SP, Ilsley D, Richardson J, Sabanathan S. A thermographic study of paravertebral analgesia. Anaesthesia. 1995;50(2):118-21. https://doi.org/10.1111/j.1365-2044.1995.tb15092.x.

26. Karmakar MK, Chui PT, Joynt GM, Ho AM. Thoracic paravertebral block for management of pain associated with multiple fractured ribs in patients with concomitant lumbar spinal trauma. Reg Anesth Pain Med. 2001;26(2):169-73. https://doi.org/10. 1053/rapm.2001.21086.

27. Ho AM, Karmakar MK, Critchley LA. Acute pain management of patients with multiple fractured ribs: a focus on regional techniques. Curr Opin Crit Care. 2011;17(4):323-7. https://doi.org/10.1097/ MCC.0b013e328348bf6f.

28. Karmakar MK. Thoracic paravertebral block. Anesthesiology. 2001;95(3):771-80. https://doi.org/10.1097/00000542200109000-00033.

29. Richardson J, Lonnqvist PA. Thoracic paravertebral block. Br J Anaesth. 1998;81(2):230-8. https://doi.org/10.1093/bja/81.2.230.

30. Mohta M, Verma P, Saxena AK, Sethi AK, Tyagi A, Girotra G. Prospective, randomized comparison of continuous thoracic epidural and thoracic paravertebral infusion in patients with unilateral multiple fractured ribs-a pilot study. J Trauma. 2009;66(4):1096101. https://doi.org/10.1097/TA.0b013e318166d76d.

31. Yeying G, Liyong Y, Yuebo C, Yu Z, Guangao Y, Weihu M, et al. Thoracic paravertebral block versus intravenous patient-controlled analgesia for pain treatment in patients with multiple rib fractures. J Int Med Res. 2017;45(6):2085-91. https://doi.org/10.1177/ 0300060517710068 Randomized trial suggesting improved ventilatory outcomes and lung mechanics in patients managed with paravertebral block.

32. Lonnqvist PA, MacKenzie J, Soni AK, Conacher ID. Paravertebral blockade. Failure rate and complications. Anaesthesia. 1995;50(9): 813-5. https://doi.org/10.1111/j.1365-2044.1995.tb06148.x.

33. Shanti CM, Carlin AM, Tyburski JG. Incidence of pneumothorax from intercostal nerve block for analgesia in rib fractures. J Trauma. 2001;51(3):536-9. https://doi.org/10.1097/00005373-20010900000019 .

34. Truitt MS, Murry J, Amos J, Lorenzo M, Mangram A, Dunn E, et al. Continuous intercostal nerve blockade for rib fractures: ready for primetime? J Trauma. 2011;71(6):1548-52; discussion 52. https://doi.org/10.1097/TA.0b013e31823c96e0.

35. Rose P, Ramlogan R, Sullivan T, Lui A. Serratus anterior plane blocks provide opioid-sparing analgesia in patients with isolated posterior rib fractures: a case series. Can J Anaesth. 2019;66(10): 1263-4. https://doi.org/10.1007/s12630-019-01431-7.

36. Southgate SJ, Herbst MK. Ultrasound guided serratus anterior blocks. Treasure Island: StatPearls; 2019.

37. Kunhabdulla NP, Agarwal A, Gaur A, Gautam SK, Gupta R, Agarwal A. Serratus anterior plane block for multiple rib fractures. Pain Phys. 2014;17(5):E651-3.

38. Forero M, Adhikary SD, Lopez H, Tsui C, Chin KJ. The erector Spinae plane block: a novel analgesic technique in thoracic neuropathic pain. Reg Anesth Pain Med. 2016;41(5):621-7. https://doi. org/10.1097/AAP.0000000000000451.

39. Klesius L, Schroeder K. Effective analgesia with bilateral erector spinae plane catheters for a patient with traumatic rib and spine 
fractures. Case Rep Anesthesiol. 2019;2019:9159878. https://doi. org/10.1155/2019/9159878.

40. Luftig J, Mantuani D, Herring AA, Dixon B, Clattenburg E, Nagdev A. Successful emergency pain control for posterior rib fractures with ultrasound-guided erector spinae plane block. Am J Emerg Med. 2018;36(8):1391-6. https://doi.org/10.1016/j.ajem. 2017.12.060.

41. Hamilton DL, Manickam B. Erector spinae plane block for pain relief in rib fractures. Br J Anaesth. 2017;118(3):474-5. https:// doi.org/10.1093/bja/aex013.

42. Adhikary SD, Liu WM, Fuller E, Cruz-Eng H, Chin KJ. The effect of erector spinae plane block on respiratory and analgesic outcomes in multiple rib fractures: a retrospective cohort study. Anaesthesia. 2019;74(5):585-93. https://doi.org/10.1111/anae.14579 Retrospective cohort study suggesting improved incentive spirometry volumes and pain scores with erector spinae block.

43. Bemelman M, Poeze M, Blokhuis TJ, Leenen LP. Historic overview of treatment techniques for rib fractures and flail chest. Eur $\mathbf{J}$ Trauma Emerg Surg. 2010;36(5):407-15. https://doi.org/10.1007/ s00068-010-0046-5.

44. Granetzny A, Abd El-Aal M, Emam E, Shalaby A, Boseila A. Surgical versus conservative treatment of flail chest. Evaluation of the pulmonary status. Interact Cardiovasc Thorac Surg. 2005;4(6): 583-7. https://doi.org/10.1510/icvts.2005.111807.

45. Marasco SF, Davies AR, Cooper J, Varma D, Bennett V, Nevill R, et al. Prospective randomized controlled trial of operative rib fixation in traumatic flail chest. J Am Coll Surg. 2013;216(5):924-32. https://doi.org/10.1016/j.jamcollsurg.2012.12.024.

46. Kasotakis G, Hasenboehler EA, Streib EW, Patel N, Patel MB, Alarcon L, et al. Operative fixation of rib fractures after blunt trauma: a practice management guideline from the Eastern Association for the Surgery of Trauma. J Trauma Acute Care Surg. 2017;82(3): 618-26. https://doi.org/10.1097/TA.0000000000001350 Recent society guidance on the role of operative fixation of rib fractures.

47. Pieracci FM, Coleman J, Ali-Osman F, Mangram A, Majercik S, White TW, et al. A multicenter evaluation of the optimal timing of surgical stabilization of rib fractures. J Trauma Acute Care Surg. 2018;84(1):1-10. https://doi.org/10.1097/TA.0000000000001729 Supports earlier surgery if rib fixation is going to be undertaken, with best outcomes in the first 24 hours.

48. Mowery NT, Gunter OL, Collier BR, Diaz JJ Jr, Haut E, Hildreth A, et al. Practice management guidelines for management of hemothorax and occult pneumothorax. J Trauma. 2011;70(2):510-8. https:// doi.org/10.1097/TA.0b013e31820b5c31.

49. Moore FO, Goslar PW, Coimbra R, Velmahos G, Brown CV, Coopwood TB Jr, et al. Blunt traumatic occult pneumothorax: is observation safe?-results of a prospective, AAST multicenter study. J Trauma. 2011;70(5):1019-23; discussion 23-5. https:// doi.org/10.1097/TA.0b013e318213f727.

50. Staub LJ, Biscaro RRM, Kaszubowski E, Maurici R. Chest ultrasonography for the emergency diagnosis of traumatic pneumothorax and haemothorax: a systematic review and meta-analysis. Injury. 2018;49(3):457-66. https://doi.org/10.1016/j.injury.2018. 01.033 .
51. Zengerink I, Brink PR, Laupland KB, Raber EL, Zygun D, Kortbeek JB. Needle thoracostomy in the treatment of a tension pneumothorax in trauma patients: what size needle? J Trauma. 2008;64(1):111-4. https://doi.org/10.1097/01.ta.0000239241. 59283.03 .

52. Inaba $\mathrm{K}$, Ives $\mathrm{C}$, McClure $\mathrm{K}$, Branco $\mathrm{BC}$, Eckstein $\mathrm{M}$, Shatz $\mathrm{D}$, et al. Radiologic evaluation of alternative sites for needle decompression of tension pneumothorax. Arch Surg. 2012;147(9):813-8. https://doi.org/10.1001/archsurg.2012.751.

53. Inaba K, Karamanos E, Skiada D, Grabo D, Hammer P, Martin M, et al. Cadaveric comparison of the optimal site for needle decompression of tension pneumothorax by prehospital care providers. J Trauma Acute Care Surg. 2015;79(6):1044-8. https://doi.org/10. 1097/TA.0000000000000849.

54. American College of S, Committee on T. Advanced trauma life support : student course manual. Chicago, IL: American College of Surgeons; 2018.

55. de Lesquen H, Avaro JP, Gust L, Ford RM, Beranger F, Natale C, et al. Surgical management for the first $48 \mathrm{~h}$ following blunt chest trauma: state of the art (excluding vascular injuries). Interact Cardiovasc Thorac Surg. 2015;20(3):399-408. https://doi.org/10. 1093/icvts/ivu397.

56.• Spahn DR, Bouillon B, Cerny V, Duranteau J, Filipescu D, Hunt $\mathrm{BJ}$, et al. The European guideline on management of major bleeding and coagulopathy following trauma: fifth edition. Crit Care. 2019;23(1):98. https://doi.org/10.1186/s13054-019-2347-3 Excellent comprehensive review of the current understanding of managing bleeding and coagulopathy of trauma.

57. Fox N, Schwartz D, Salazar JH, Haut ER, Dahm P, Black JH, et al. Evaluation and management of blunt traumatic aortic injury: a practice management guideline from the Eastern Association for the Surgery of Trauma. J Trauma Nurs. 2015;22(2):99-110. https:// doi.org/10.1097/JTN.0000000000000118.

58. Scalea TM, Feliciano DV, DuBose JJ, Ottochian M, O'Connor JV, Morrison JJ. Blunt thoracic aortic injury: endovascular repair is now the standard. J Am Coll Surg. 2019;228(4):605-10. https:// doi.org/10.1016/j.jamcollsurg.2018.12.022.

59. Hanschen M, Kanz KG, Kirchhoff C, Khalil PN, Wierer M, van Griensven M, et al. Blunt cardiac injury in the severely injured - a retrospective multicentre study. PLoS One. 2015;10(7):e0131362. https://doi.org/10.1371/journal.pone.0131362.

60. Nagy KK, Krosner SM, Roberts RR, Joseph KT, Smith RF, Barrett J. Determining which patients require evaluation for blunt cardiac injury following blunt chest trauma. World J Surg. 2001;25(1):108 11. https://doi.org/10.1007/s002680020372.

61. Joseph B, Jokar TO, Khalil M, Haider AA, Kulvatunyou N, Zangbar B, et al. Identifying the broken heart: predictors of mortality and morbidity in suspected blunt cardiac injury. Am J Surg. 2016;211(6):982-8. https://doi.org/10.1016/j.amjsurg.2015.10.027 Excellent summary of the risk factors for poor outcome in blunt cardiac injury.

Publisher's Note Springer Nature remains neutral with regard to jurisdictional claims in published maps and institutional affiliations. 